Research Article

\title{
Improved Strategy of Two-Node Curved Beam Element Based on the Same Beam's Nodes Information
}

\author{
Yuquan Wang (1) \\ China Railway Design Corporation, Tianjin 300308, China \\ Correspondence should be addressed to Yuquan Wang; wangyuquan@crdc.com
}

Received 14 May 2021; Revised 16 August 2021; Accepted 17 August 2021; Published 2 September 2021

Academic Editor: Angela De Bonis

Copyright (c) 2021 Yuquan Wang. This is an open access article distributed under the Creative Commons Attribution License, which permits unrestricted use, distribution, and reproduction in any medium, provided the original work is properly cited.

\begin{abstract}
The curved beam with a great initial curvature is the typical structure and applied widely in real engineering structures. The common practice in the current literature employs two-node straight beam elements as the elementary members for stress and displacement analysis, which needs a large number of divisions to fit the curved beam shape well and increases computational time greatly. In this paper, we develop an improved accurate two-node curved beam element (IC2) in 3D problems, combining the curved Timoshenko beam theory and the curvature information calculated from the same beam curve. The strategy of calculating the curvature information from the same bean curve in the IC2 beam element and then transferring the curvature information to the two-node straight beam element can greatly enhance the accuracy of the mechanical analysis with no extra calculation burden. We then introduce the finite element implementation of the IC2 beam element and verify by the complex curved beam analysis. By comparison with simulation results from the straight two-node beam element in the MIDAS (S2-MIDAS) and the three-node curved beam element adopted in the ANSYS (C3-ANSYS), the simulation results of the typical quarter arc examples under constant or variable curvature show that the IC2 beam element based on curved beam theory is a combination of efficiency and accuracy. And, it is a good choice for analysis of complex engineering rod structure with large initial curvature.
\end{abstract}

\section{Introduction}

The curved beam is a typical structure model and applied widely in real engineering structures, such as bridges and train tracks. Finite element (FE) method based on the beam deformation theory is a very efficient method to its mechanical analysis of any complex beam structures [1-5]. A usual strategy of the finite element analysis of the rod with large initial curvature (as shown in Figure 1(a)), which has been applied widely in the engineering analysis and design software package (such as ANSYS and MIDAS), is using a large number of straight elements to approximate its true curve shape and ensure analysis precisions, as shown in Figure 1(b) [6-10]. However, there needs to be a large number of divisions to fit the curved beam shape well, which increases computational time greatly, especially for the curved beam with great initial curvature. For better efficiency, there is focus on developing the curved beam theory and the related special elements, which contain the influence of the curvature information for curved rods structures. For example, in Figure 1(c), we can see that the curved beam element can fit the curved beam curve better than the straight beam element under the same number of divisions [11, 12].

In order to calculate the curvature information of the curved beam element, a common strategy is to develop higher order curved beam element to calculate the curvature information in the inner regions of the beam [12-17]. Taking the three-node curved beam element as an example, adapted in the professional finite element software ANSYS (C3-ANSYS), three nodes in an element do not have to be colinear, and the curvature information in the element can be calculated by nodes interpolation method. However, the adding middle points increase element nodes and calculation costs, and also the curvature information, calculated based on the local region information, can cause great errors when the beam curve is variable curvature with great change rate $[18,19]$.

Over these considerations, the two-node general curved beam element is the best choice of a combination of 


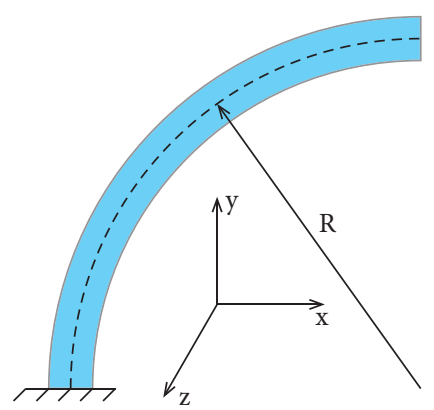

(a)

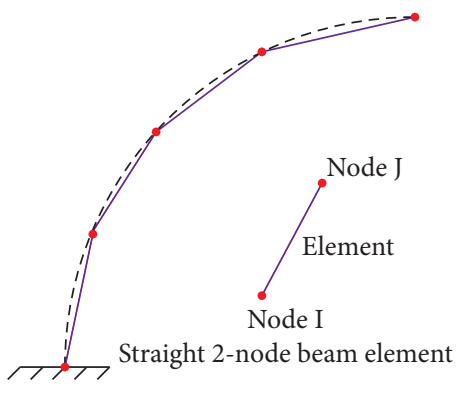

(b)

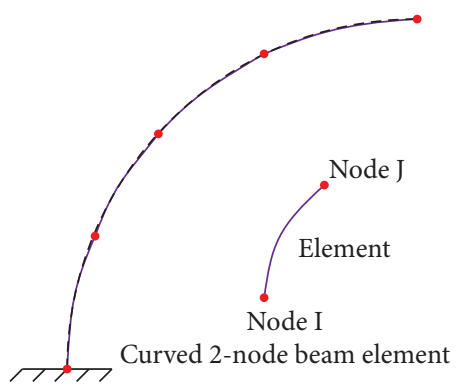

(c)

FIgURE 1: Schematic illustrations of a typical three-dimensional curved beam. (a) A quarter arc curved beam with radius $R$, divided by (b) the straight two-node beam element and (c) the curved two-node beam element.

efficiency and accuracy for mechanical analysis based on the FE method [20-22]. For example, the study in [23] derived exact shape functions for a two-node uniform circular beam element with constant curvature; the study in [24] developed the two-node curved element and applied FE analysis of the curved beam structures. In order to enhance the efficiency and accuracy by decreasing the divided element number in initially curved beams with large deflections, the study in [25] introduced the scheme of the unequal discretization and applied for compliant mechanisms [26]. A major problem in the initial curved beam with great curvature change rate is the calculated strategy on the curvature of the $\mathrm{C} 2$ beam element with the lack of curvature information and then greatly affecting the accuracy in the analysis of the initially curved beam structures.

In this paper, we developed an improved strategy of twonode curved beam element based on the neighbor or the whole beam nodes information for the analysis of the threedimensional curved beam structure problems. In Section 2, a detailed discussion of the curved beam theory and its finite element formulation are introduced. Also, two types of curvature information calculation strategy of the improved C2 (IC2) beam element are introduced. In Section 3, the verifications of the quarter arc model with constant curvature and the elliptical shape quarter arc beam model are demonstrated. Finally, the conclusion is in Section 4.

\section{Three-Dimensional Curved Beam Theory and Its Finite Element Formulation}

In the section, we firstly summarize the basic three-dimensional curved beam theory which has been presented by Reissner [27]. And then, the finite element formulation of Reissner's curved beam theory and its variational equations are introduced.

2.1. Basic Equations. Before we introduce the basic equations, we firstly introduce two coordinate systems. As shown in Figure 2(a), the $x$-, $y$-, and $z$-directions are defined in the global coordinate system, which is fixed and independent with the location $s$ in the axis of the beam. In contrast, the local coordinate system, that is, $x^{\prime}-, y^{\prime}$-, and $z^{\prime}$-directions, is defined in the axis of the beam and changes as the position $s \in[0, L]$, which denotes the curvilinear coordinate along the axis of the curved beam. Moreover, the $x^{\prime}$-direction and $y^{\prime}$-direction are the cross-sectional coordinate system, and $z^{\prime}$-direction is along the direction of the beam axis.

As shown in Figure 2(a), the equilibrium equations in the global coordinate system, considering the stress resultants and couples parameterized over a curved beam reference configuration, can be written as

$$
\begin{array}{r}
\frac{\mathrm{d} \mathbf{n}}{\mathrm{d} s}+\bar{m}=0, \\
\frac{\mathrm{d} \mathbf{m}}{\mathrm{d} s}+\frac{\mathrm{d} \phi}{\mathrm{d} s} \times \mathbf{n}+m \bar{m}=0,
\end{array}
$$

where $\mathbf{n}=\left(n_{x}, n_{y}, n_{z}\right)^{T}$ and $\overline{\mathbf{n}}=\left(\bar{n}_{x}, \bar{n}_{y}, \bar{n}_{z}\right)^{T}$ are the stress resultants vector and the outer load vector, respectively, $\mathbf{m}=$ $\left(m_{x}, m_{y}, m_{z}\right)^{T}$ and $\overline{\mathbf{m}}=\left(\bar{m}_{x}, \bar{m}_{y}, \bar{m}_{z}\right)^{T}$ are the stress couples and the outer couples load vector, respectively, and $(\mathrm{d} \phi / \mathrm{d} s) \times$ is the skew symmetric matrix associated with the map $\phi=$ $((\mathrm{d} x / \mathrm{d} s),(\mathrm{d} y / \mathrm{d} s),(\mathrm{d} z / \mathrm{d} s))$ as

$$
\frac{\mathrm{d} \phi}{\mathrm{d} s} \times=\left[\begin{array}{ccc}
0 & -\frac{\mathrm{d} z}{\mathrm{~d} s} & \frac{\mathrm{d} y}{\mathrm{~d} s} \\
\frac{\mathrm{d} z}{\mathrm{~d} s} & 0 & -\frac{\mathrm{d} x}{\mathrm{~d} s} \\
-\frac{\mathrm{d} y}{\mathrm{~d} s} & \frac{\mathrm{d} x}{\mathrm{~d} s} & 0
\end{array}\right],
$$

which contains the curvature information of the curved axis of the beam. When the axis of the beam is straight and its direction coincides with the $z$-axial direction, then there is $(\mathrm{d} z / \mathrm{d} s)=1,(\mathrm{~d} x / \mathrm{d} s)=(\mathrm{d} y / \mathrm{d} s)=0$ and the equivalent equations of the resultant force and couples can be given as

$$
\begin{aligned}
& \frac{\mathrm{d} n_{x}}{\mathrm{~d} z}+\bar{n}_{x}=0, \\
& \frac{\mathrm{d} n_{y}}{\mathrm{~d} z}+\bar{n}_{y}=0, \\
& \frac{\mathrm{d} n_{z}}{\mathrm{~d} z}+\bar{n}_{z}=0,
\end{aligned}
$$




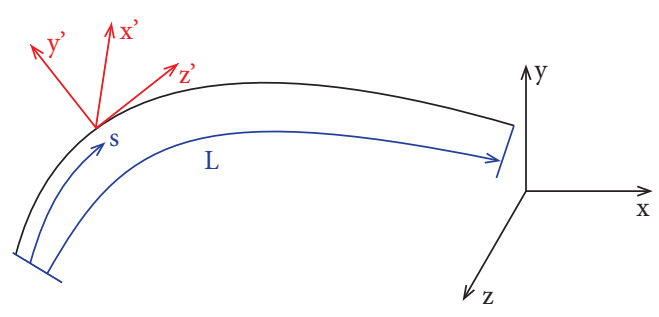

(a)

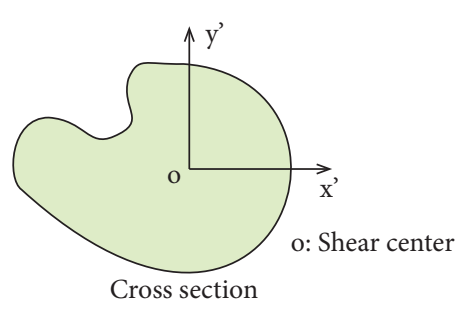

(b)

FIGURE 2: Illustrations of the coordinate systems for (a) an arbitrary curved beam and (b) its cross section.

$$
\begin{array}{r}
\frac{\mathrm{d} m_{x}}{\mathrm{~d} z}-n_{y}+\bar{m}_{x}=0, \\
\frac{\mathrm{d} m_{y}}{\mathrm{~d} z}+n_{x}+\bar{m}_{t}=0, \\
\frac{\mathrm{d} m_{z}}{\mathrm{~d} z}+\bar{m}_{z}=0 .
\end{array}
$$

The equations in (3a) and (3b) are the equilibrium equations on the typical straight beam theory [28].

Following Reissner's curved beam theory, the strain measures, which are conjugated to the stress resultants and couples, are given as

$$
\left(\widehat{\boldsymbol{\varepsilon}}^{\prime}\right)=\left(\frac{\partial u^{\prime}}{\partial z^{\prime}}-\theta_{y^{\prime}}, \frac{\partial v^{\prime}}{\partial z^{\prime}}+\theta_{x^{\prime}}, \frac{\partial w^{\prime}}{\partial z^{\prime}}, \frac{\partial \theta_{x^{\prime}}}{\partial z^{\prime}}, \frac{\partial \theta_{y^{\prime}}}{\partial z^{\prime}}, \frac{\partial \theta_{z^{\prime}}}{\partial z^{\prime}}\right)^{T}
$$

where the superscript / represents that the variable is located in the local coordinate system; symbols $u^{\prime}, v^{\prime}, w^{\prime}$, defined in the local coordinate system, are displacement variables in the $x^{\prime}-, \quad y^{\prime}-$, and $z^{\prime}$-directions, respectively; and symbols $\theta_{x^{\prime}}, \theta_{y^{\prime}}, \theta_{z^{\prime}}$ are rotation variables, respectively.

In the paper, we assume that the properties of the beam's material are isotropic and elastic. Then, the generalized constitutive matrix for descriptions of the relationship between the stress resultants and moments vector and the strain vector are constant and diagonal and given as

$$
[\widehat{\mathbf{D}}]=\operatorname{diag}\left(k_{x^{\prime}} G A, k_{y^{\prime}} G A, E A, E I_{x^{\prime}}, E I_{y^{\prime}}, G J\right) \text {, }
$$

where $k_{x^{\prime}} G A$ and $k_{y^{\prime}} G A$ are the shear stiffness along axes $x^{\prime}$ and $y^{\prime}, k_{x^{\prime}}$ and $k_{y^{\prime}}$ are the shear factors, $E A$ is the axial stiffness, $E I_{x^{\prime}}$ and $E I_{y^{\prime}}$ are the principal bending stiffnesses relative, and $G J$ is the torsional stiffness.

2.2. Finite Element Approximation. In the previous section, we introduced the complete summary of the curved beam theory and its basic equations, which are considered in this paper. Now let us introduce its finite element implementations of the curved beam theory to the IC2 beam element in detail.

Considering a standard beam curve with the length $L$ (as shown in Figure 2(a)), we can obtain the virtual work equations according to (1) in the form

$$
\delta \Pi=\int_{L}\left[\delta \mathbf{u}\left(\frac{\mathrm{d} \mathbf{n}}{\mathrm{d} s}+\bar{n}\right)+\delta \vartheta\left(\frac{\mathrm{d} \mathbf{m}}{\mathrm{d} s}+\frac{\mathrm{d} \phi}{\mathrm{d} s} \times \mathbf{n}+\bar{m}\right)\right] \mathrm{d} s=0,
$$

where $\delta \mathbf{u}$ and $\delta \vartheta$ are the virtual displacements vector and the virtual rotations vector. Also, the virtual work equation can be retreated as

$$
\delta \Pi=\int_{L} \delta \widehat{\boldsymbol{\varepsilon}}^{T}(\mathbf{n} ; \mathbf{m}) \mathrm{d} s-\int_{L}[\delta \mathbf{u} \cdot \overline{\mathbf{n}}+\delta \vartheta \cdot \overline{\mathbf{m}}] \mathrm{d} s,
$$

where

$\delta \widehat{\varepsilon}=(\delta \epsilon ; \delta \kappa), \delta \epsilon=(\mathrm{d} \delta \mathbf{u} / \mathrm{d} s)-\delta \vartheta \times(\mathrm{d} \phi / \mathrm{d} s), \delta \kappa=(\mathrm{d} \delta \vartheta / \mathrm{d} s)$, and $\delta \epsilon$ and $\delta \kappa$ are the virtual strain measures energy-conjugate to stress results $\mathbf{n}$ and couples $\mathbf{m}$. Combined with (4)-(6), there is the tangent operator of the virtual work equations (in (7))

$$
d \delta \Pi=\int_{L}\left([\mathbf{B}]\left[\begin{array}{cc}
\boldsymbol{\Lambda}^{\mathbf{T}} & 0 \\
0 & \boldsymbol{\Lambda}^{\mathbf{T}}
\end{array}\right](\boldsymbol{\delta} \widehat{u})\right)^{T}[\widehat{D}][\mathbf{B}]\left[\begin{array}{cc}
\boldsymbol{\Lambda}^{\mathbf{T}} & \mathbf{0} \\
\mathbf{0} & \boldsymbol{\Lambda}^{\mathbf{T}}
\end{array}\right](\mathrm{d} \widehat{u}) \mathrm{d} s,
$$

where the matrix $[\Lambda]$ is the transformation matrix from the global coordinate system to the local coordinate system (as shown in Figure 2(a)), which is written as

$$
[\boldsymbol{\Lambda}]_{3 \times 3}=\left[\begin{array}{lll}
\frac{\mathrm{d} x^{\prime}}{\mathrm{d} x} & \frac{\mathrm{d} y^{\prime}}{\mathrm{d} x} & \frac{\mathrm{d} z^{\prime}}{\mathrm{d} x} \\
\frac{\mathrm{d} x^{\prime}}{\mathrm{d} y} & \frac{\mathrm{d} y^{\prime}}{\mathrm{d} y} & \frac{\mathrm{d} z^{\prime}}{\mathrm{d} y} \\
\frac{\mathrm{d} x^{\prime}}{\mathrm{d} z} & \frac{\mathrm{d} y^{\prime}}{\mathrm{d} z} & \frac{\mathrm{d} z^{\prime}}{\mathrm{d} z}
\end{array}\right] .
$$

And, the matrix $[\mathbf{B}]$ is defined as

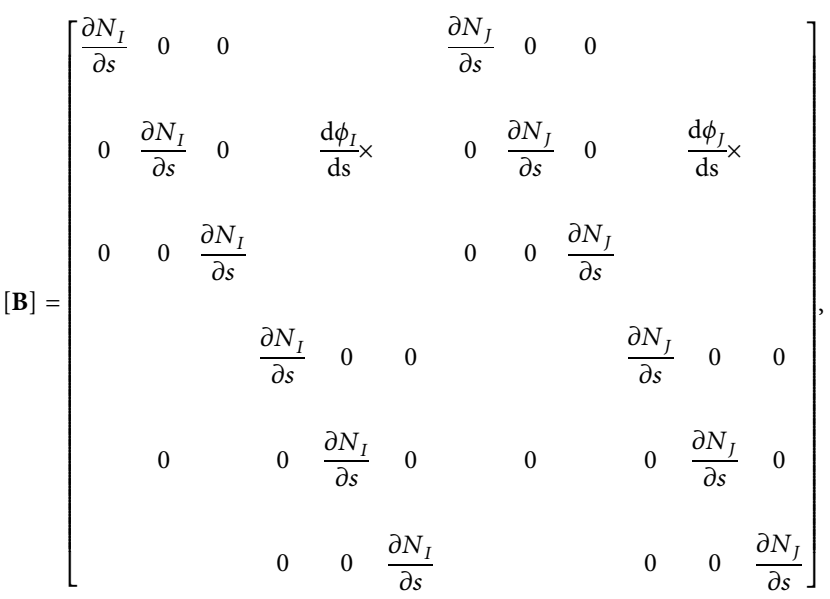


which contains curvature information of the curved beam element. For the IC2 beam element, uniformly reduced integrated form [29] and linear interpolation functions of the displacements and rotations vectors [30] are applied for the linear problems. So, here we assume that the variable $a$ represents displacements, rotations, or other variables, and it can be interpolated as

$$
a=N_{I} a_{I}+N_{J} a_{J}
$$

where $I$ and $J$ denote nodes' number at both ends of the IC2 beam element (as shown in Figure 1(c)) and $N_{I}$ and $N_{J}$ are the corresponding interpolation functions, which can be expressed as $N_{I}=(s / L), N_{J}=1-(s / L)$.

2.3. Curvature Information Calculation. Curvature information, which has been used in the curved beam theory, can enhance the accuracy of the numerical results compared with the straight beam element based on the straight beam theory. So, the accuracy of the curvature information, which is calculated and passed to the IC2 beam element, is extremely important for precisions of the numerical results of rods. The common curvature information calculation strategy is by adding middle points at the inner part of the element in the curved element. However, the added middle nodes increase the calculation costs, and the curvature information can only reflect on the local information and may cause great errors for rods with variable curvature. In the paper, we consider two types of curvature information calculation schemes to eliminate the above two problems. Scheme A: curvature information can be calculated based on the neighbor two nodes' coordinate information of the same beam. Hence, a cubic interpolation function is used for fitting shapes of the IC2 beam element as

$$
\begin{aligned}
& N_{1}=\frac{1}{16}\left[-9\left(\frac{s}{L}\right)^{3}+9\left(\frac{s}{L}\right)^{2}+\left(\frac{s}{L}\right)^{1}-1\right] \\
& N_{2}=\frac{1}{16}\left[9\left(\frac{s}{L}\right)^{3}+9\left(\frac{s}{L}\right)^{2}-\left(\frac{s}{L}\right)^{1}-1\right], \\
& N_{3}=\frac{1}{16}\left[27\left(\frac{s}{L}\right)^{3}-9\left(\frac{s}{L}\right)^{2}-27\left(\frac{s}{L}\right)^{1}+9\right] \\
& N_{4}=\frac{1}{16}\left[-27\left(\frac{s}{L}\right)^{3}-9\left(\frac{s}{L}\right)^{2}-27\left(\frac{s}{L}\right)^{1}+9\right]
\end{aligned}
$$

and scheme B: we precalculate and save the curvature of the whole beam before we start to the numerical analysis of the complex rod structure and then pass the curvature information to the IC2 beam element to start the mechanical analysis of the beam structure by the FE numerical calculations.

\section{Numerical Simulations}

In this section, we will verify the accuracy of the IC2 beam element for numerical results of complex curved beam structures by comparison with the S2-MIDAS beam element and the C3-ANSYS beam element. Herein, the S2-MIDAS beam element is developed based on the straight beam theory and has been integrated in the MIDAS software. And the C3ANSYS beam element based on the curved beam theory has been developed for the curved beam analysis, which contains curvature information by adding midnode. In order to verify the accuracy and efficiency of the analysis method with the IC2 element, we show the displacement and force field simulation results of a quarter arc example by comparison with the calculation results from the S2-MIDAS beam element in the MIDAS software and the C3-ANSYS beam element.

\subsection{Test Case 1: A Quarter Arc Beam Model with Constant} Curvature. Firstly, we consider a typical curved beam example of a quarter arc beam model (as shown in Figure 3(a)), which is fixed at the bottom end and loaded at the top end. And the displacement and rotation constraints at the top end are $\Delta u_{x}=0.1 m, \Delta_{y}=-0.1 m, \Delta \theta_{x}=0.1 \mathrm{rad}, \Delta \theta_{z}=-0.1 \mathrm{rad}$.

Here the quarter arc beam's curvature radius is $R=1 \mathrm{~m}$, and the circular cross section's radius is $r=0.1 \mathrm{~m}$. The cross section's materials are isotropic and elastic as Young's modulus $E=210 \mathrm{GPa}$ and Poisson's ratio $\nu=0.3$, respectively. The $3 \mathrm{D}$ solid finite element model in Figure 3(a) is also worked to verify the calculated force and moments results based on the beam element model. With consideration of the quarter beam with constant curvature along the beam axis, here we take scheme A as the applied strategy for calculating the curvature information from the neighbor nodes in the IC2 beam element.

Table 1 lists the numerical results of $u_{z}$ and the displacement in the $z$-direction at the top end of the quarter arc beam under the influence of the no. of divisions. In Figure $4(\mathrm{a})$, we can see that $u_{z}$ decreases and gradually converges to a constant solution as the no. of divisions increases. For the numerical results from the C3-ANSYS beam element, there is the highest converge rate by comparison with that from the IC2 beam element and the S2MIDAS beam element, and we can see that only two divisions can converge to the constant value, that is, $u_{z}=0.0280 \mathrm{~m}$. Taking the solution as the stable value, we can see that the numerical results from the IC2 beam element can converge to the stable value more quickly than that from the S2-MIDAS element in Figure 4(a), as divisions of the quarter arc beam increase. Also, we list and plot the numerical results of the rotation $\theta_{y}$ in the $y$-direction at the top end of the curved beam (as shown in Table 2 and Figure 4(b)). Similarly, the numerical results from the C3ANSYS beam element, which has the highest converge rate, converge to the stable value, $\theta_{y}=0.00830 \mathrm{rad}$, by two divisions. For the behavior of the IC2 beam element, we can see that three divisions by the IC2 element type can get the closer stable solutions, $\theta_{y}=0.00833 \mathrm{rad}$. Meanwhile, there needs to be 10 more divisions of S2-MIDAS beam element type to converge to the stable solution, as shown in Figure 4(b). That is to say, the IC2 beam element can get greater accuracy of the curved beam structure's numerical results than that of the S2-MIDAS beam element based on the straight beam theory, while the C3-ANSYS beam element has the best accuracy and converge rate of the 


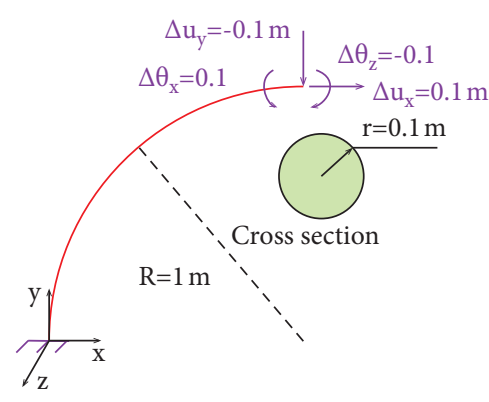

(a)

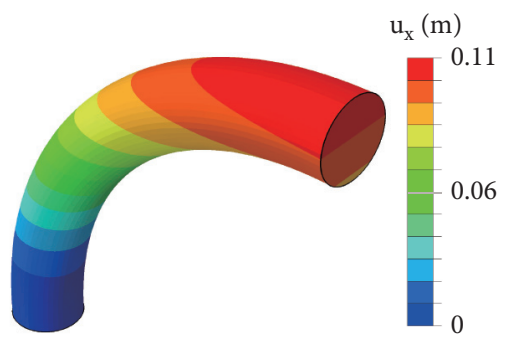

(c)

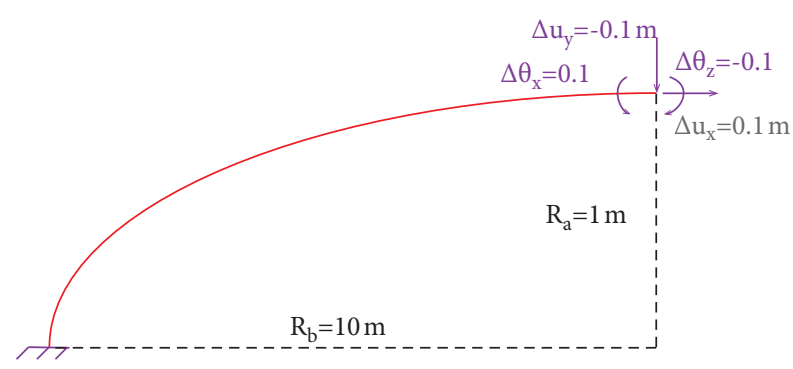

(b)

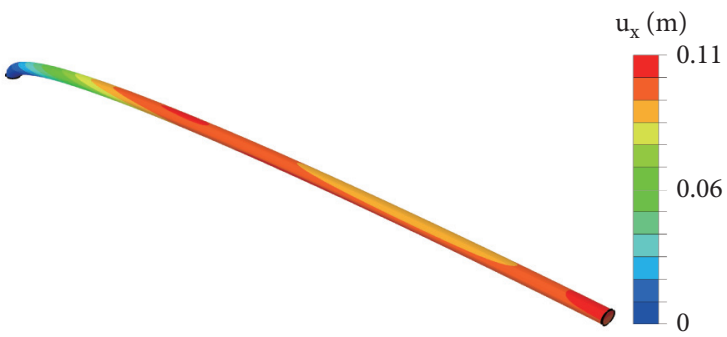

(d)

Figure 3: The quarter arc beam model of (a) the circular shape and (b) the elliptical shape. (c, d) Its cross section's shape is circular, and its corresponding 3D solid finite element model.

TABLE 1: $u_{z}$, the maximum displacement of the quarter arc beam in the $z$-direction.

\begin{tabular}{lccccrr}
\hline \multirow{2}{*}{ Software } & \multirow{2}{*}{ Element type } & \multicolumn{4}{c}{ No. of elements } \\
& & 2 & 3 & 5 & 0.0286 & 0.0284 \\
\hline MIDAS & S2 & 0.0318 & 0.0297 & 0.0282 & 0.0280 \\
ANSYS & C3 & 0.0280 & 0.0280 & 0.0280 & 0.0280 \\
The paper & C2 & 0.0283 & 0.0281 & 0.0280 & 0.0280 & 0.0280 \\
\hline
\end{tabular}

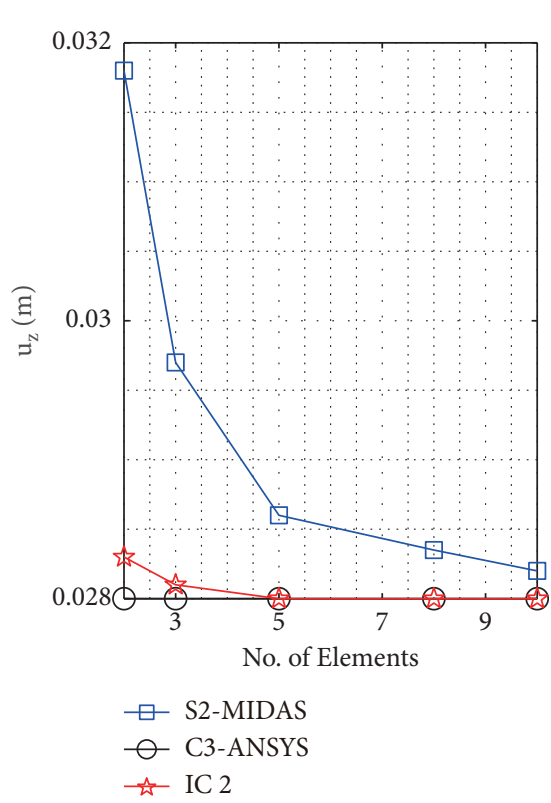

(a)

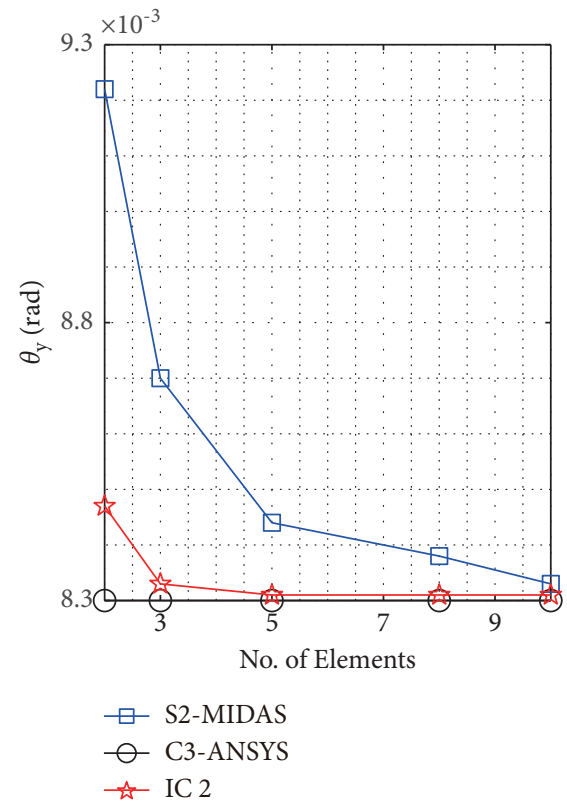

(b)

FIGURE 4: Influences of element types and no. of divisions on the numerical solutions of (a) $u_{z}$ and (b) $\theta_{y}$. 
TABLE 2: $\theta_{y}$, the maximum rotation of the quarter arc beam in the $y$-direction.

\begin{tabular}{|c|c|c|c|c|c|c|}
\hline \multirow{2}{*}{ Software } & \multirow{2}{*}{ Element type } & \multicolumn{5}{|c|}{ No. of elements } \\
\hline & & 2 & 3 & 5 & 8 & 10 \\
\hline MIDAS & $\mathrm{S} 2$ & 0.00922 & 0.00870 & 0.00844 & 0.00838 & 0.00833 \\
\hline ANSYS & $\mathrm{C} 3$ & 0.00830 & 0.00830 & 0.00830 & 0.00830 & 0.00830 \\
\hline The paper & $\mathrm{C} 2$ & 0.00847 & 0.00833 & 0.00831 & 0.00831 & 0.00831 \\
\hline
\end{tabular}

TABLE 3: The maximum stress resultants and couples of the quarter arc beam.

\begin{tabular}{|c|c|c|c|c|c|c|c|c|c|}
\hline \multirow{2}{*}{ Software } & \multirow{2}{*}{ Element type } & \multicolumn{2}{|c|}{$F_{x}$} & \multicolumn{2}{|c|}{$F_{y}$} & \multicolumn{2}{|l|}{$M_{x}$} & \multicolumn{2}{|l|}{$M_{z}$} \\
\hline & & Value (MN) & Error (\%) & Value $(\mathrm{MN})$ & Error (\%) & Value $(\mathrm{MN} \cdot \mathrm{m})$ & Error (\%) & Value $(\mathrm{MN} \cdot \mathrm{m})$ & Error (\%) \\
\hline MIDAS & S2 & -15.66 & -1.45 & -10.03 & -2.62 & -0.91 & 0 & 7.34 & -1.19 \\
\hline ANSYS & C3 & -15.88 & 0 & -10.28 & 0.19 & -0.91 & 0 & 7.42 & 0 \\
\hline The paper & $\mathrm{C} 2$ & -15.83 & -0.38 & -10.20 & -0.97 & -0.91 & 0 & 7.39 & 0.51 \\
\hline $3 \mathrm{D}$ sol & id analysis & -15.89 & 0 & -10.30 & 0 & -0.91 & 0 & 7.42 & 0 \\
\hline
\end{tabular}

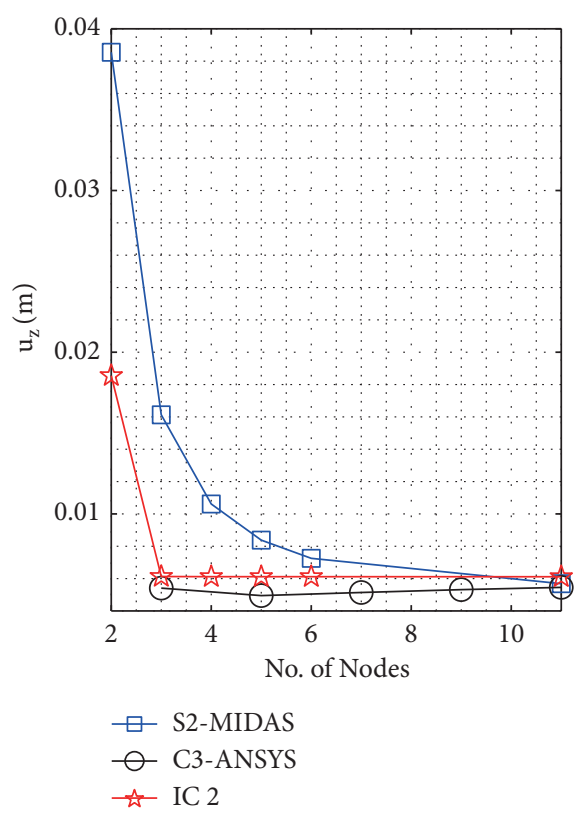

(a)

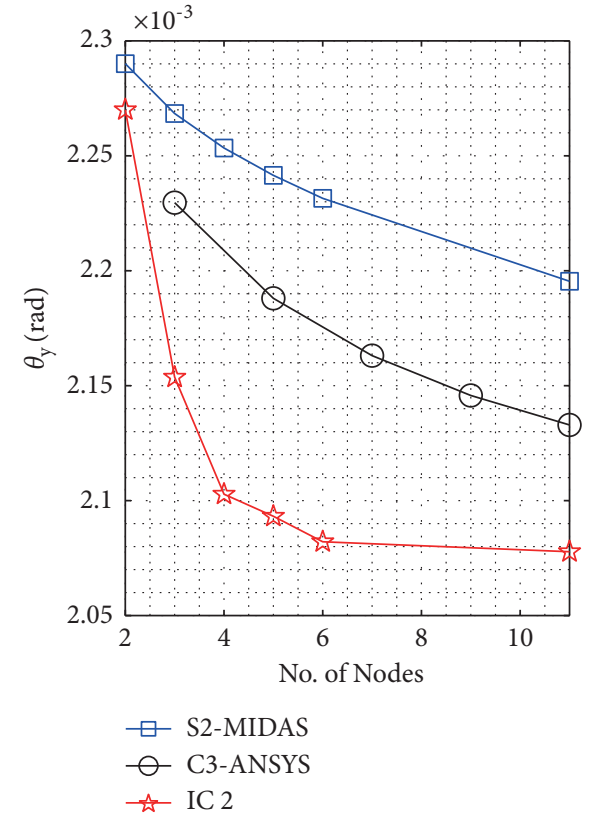

(b)

FIGURE 5: Influences of element types and no. of divided nodes on the numerical solutions of (a) $u_{z}$ and (b) $\theta_{y}$.

displacement solutions than that of the IC2 beam element, whose curvature information is calculated based on the neighbor nodes' information.

In Table 3, we list the forces, $F_{x}$ in the $x$-direction and $F_{y}$ in the $y$-direction, and the moments, $M_{x}$ in the $x$-direction and $M_{z}$ in the $z$-direction, at the bottom end of the quarter arc beam, respectively. And the quarter arc beam has been divided into 10 divisions. By comparison with the beam finite element analysis, we here also list the average force and moment at the bottom surface in Table 3, which well agrees with the numerical solutions from the C3-ANSYS beam element. Taking the numerical solutions from the $3 \mathrm{D}$ solid analysis as the stable values, we can see that the forces and moment field solutions from the IC2 element type have less errors than that from the S2-MIDAS beam element.

\subsection{Test Case 2: A Quarter Arc Beam Model with Variable} Curvature. For the curved beam with constant curvature, our results show that the IC2 beam element based on the curved beam theory and curvature information calculation scheme from neighbor nodes' interpolation method has higher precisions of numerical results than the S2-MIDAS beam element based on the straight beam theory, but not more accurate than that from the C3-ANSYS beam element. However, most of rod structures are variable curvature, and uniform divisions of the whole beam curve can cause great errors in the region with large curvature change rate (such as the bottom end of the quarter elliptical arc structure in Figure 3(b)). Here we consider a variable curvature curved beam example of a quarter arc beam model (as shown in Figure 3(b)), all but the shape is the same with Figure 3(a). 


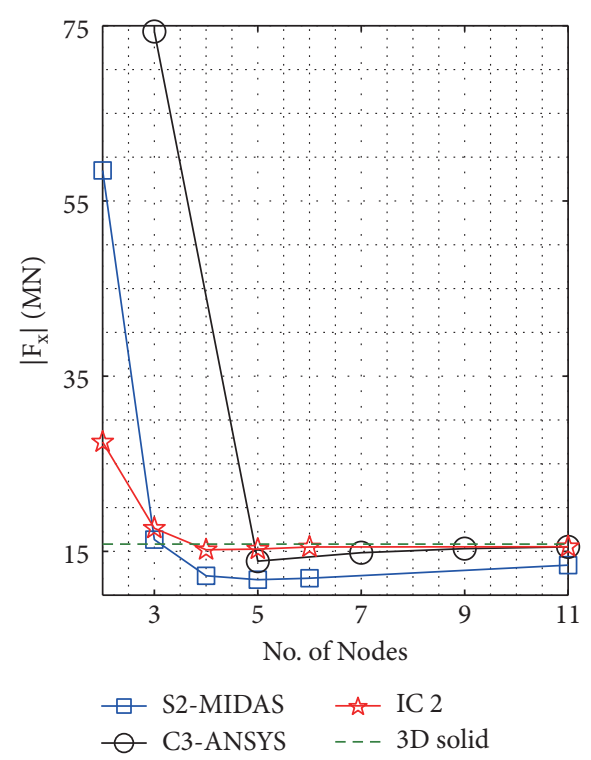

(a)

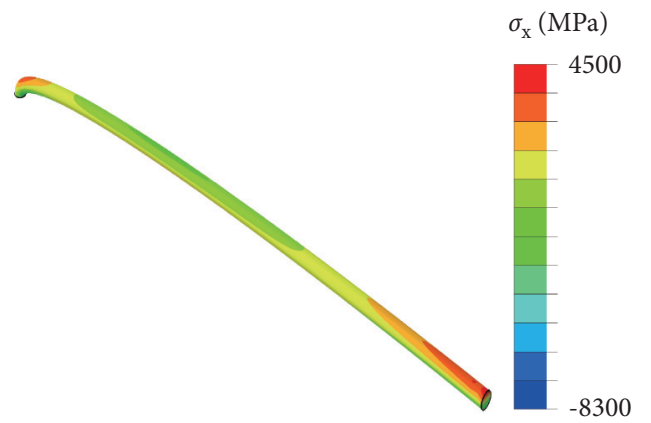

(c)

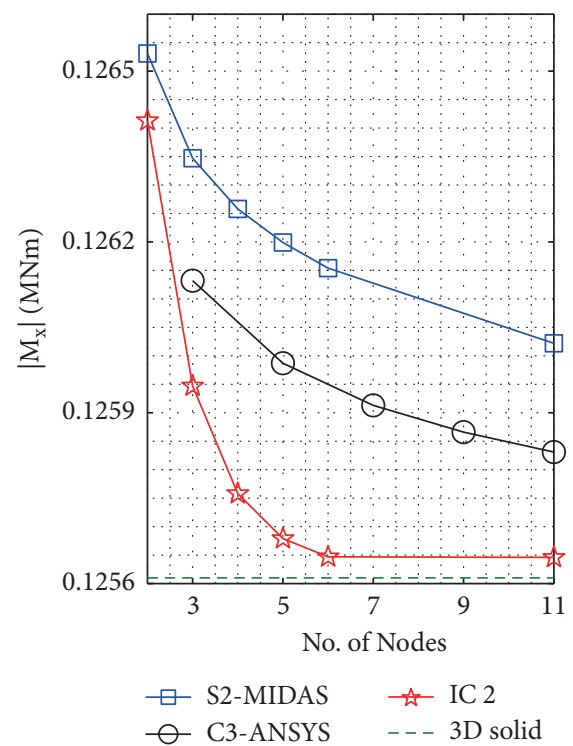

(b)

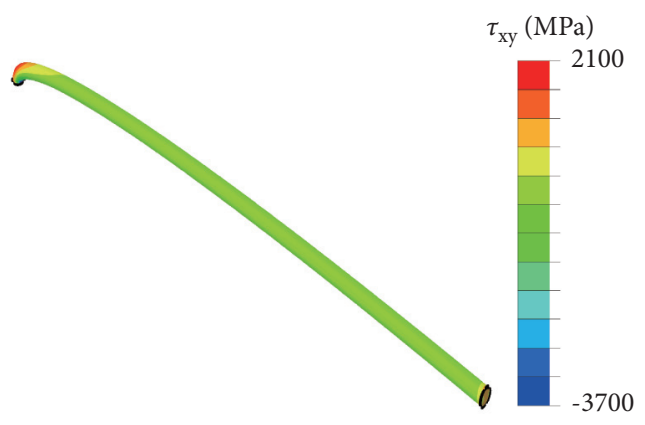

(d)

Figure 6: Influences of element types and no. of divided nodes on the numerical solutions of (a) $\left|F_{x}\right|$ and (b) $\left|M_{x}\right|$. The stress distributed contours from the $3 \mathrm{D}$ solid finite element analysis: (c) $\sigma_{x}$ and (d) $\tau_{x y}$.

Here the quarter arc beam's curvature radius is $R_{a}=1 \mathrm{~m}$ at the top end of the beam and $R_{b}=10 \mathrm{~m}$ at the bottom end of the beam. Different from the curvature information calculation scheme we adopted in test case 1, here we precalculate full curvature information along the whole beam axis and then pass the curvature information to the IC2 beam element and start to the mechanical analysis of any complex curved beam. The strategy has been applied when there is huge change rate of the curvature for the quarter elliptical arc beam model in Figure 3(b).

We firstly show in Figures 5(a) and 5(b) the influence of the no. of nodes on the displacement field solutions, the displacement $u_{z}$ in the $z$-direction, and the rotation $\theta_{y}$ in the $y$-direction at the top end of the curved beam, respectively. We can see that the displacement solutions decrease and converge to the stable values as the no. of nodes increases. By comparison with the numerical results from the C3-ANSYS beam element and the S2-MIDAS beam element in the MIDAS, there is the fastest convergence rate of $u_{z}$ and $\theta_{y}$ based on the IC2 element, and the C3-ANSYS element comes second. Here we know that the curvature information calculation strategy works for the curved beam with great change rate by improving the accuracy of the curvature information to the IC2 element. We then show in Figures 6(a) and 6(b) the forces and moment field solutions, and the numerical results from the 3D solid analysis are also given, which is extracted from the stress distributions shown in Figures 6(c) and 6(d). We can see that the IC2 element based on the curvature information calculation from the whole beam axis can increase the convergence rate and the accuracy of the solutions greatly when the beam axis has great change rate of the curvature. Also, the curvature information calculation strategy works for the numerical solutions of the force and moment fields.

\section{Conclusions}

An improved two-node curved beam element based on the curved beam theory and the curvature information calculation strategies based on the whole beam axis are proposed for analysis of the rod structure model with large initial curvature. We take a quarter arc beam model as the typical example and discuss the influence of numerical calculation results of the IC2 element on the accuracy and efficiency by 
comparison with the S2-MIDAS beam element in MIDAS and the C3-ANSYS beam element in the paper. Our results show that there needs to be fewer divisions, though achieving higher precisions of the numerical displacements and force results compared with the straight two-node element. Compared with the three-node curved beam element, there are few improvements in accuracy of the numerical results in the quarter arc beam example, which has constant curvature in the whole beam axis. However, the IC2 beam element based on the whole curvature information calculation scheme has greater improvements by comparison with the C3-ANSYS beam element when the curved beam has great change rate of the curvature. And, it is a good choice for analysis of complex engineering rod structure with large initial curvature or curvature change rate.

\section{Data Availability}

Some or all data, models, or codes that support the findings of this study are available from the corresponding author upon reasonable request.

\section{Conflicts of Interest}

The author declares that there are no conflicts of interest associated with this study.

\section{References}

[1] K. Fumio, "On the validity of the finite element analysis of circular arches represented by an assemblage of beam elements," Computer Methods in Applied Mechanics and Engineering, vol. 5, no. 3, pp. 253-276, 1975.

[2] S. P. Timoshenko, "LXVI. On the correction for shear of the differential equation for transverse vibrations of prismatic bars," The London, Edinburgh, and Dublin Philosophical Magazine and Journal of Science, vol. 41, no. 245, pp. 744-746, 1921.

[3] S. P. Timoshenko, "X. On the transverse vibrations of bars of uniform cross-section," The London, Edinburgh, and Dublin Philosophical Magazine and Journal of Science, vol. 43, no. 253, pp. 125-131, 1922.

[4] E. Oñate, Structural Analysis with the Finite Element Method, Springer, Berlin, Germany, 2009.

[5] S. Szugat, J. Heinrich, R. Maurer, and C. H. Müller, "Prediction intervals for the failure time of prestressed concrete beams," Advances in Materials Science and Engineering, vol. 2016, Article ID 9605450, 8 pages, 2016.

[6] F. Kikuchi and K. Tanizawa, "Accuracy and locking-free property of the beam element approximation for arch problems," Computers \& Structures, vol. 19, no. 1-2, pp. 103-110, 1984.

[7] B. N. Alemdar and D. W. White, "Displacement, flexibility, and mixed beam-column finite element formulations for distributed plasticity analysis," Journal of Structural Engineering, vol. 131, no. 12, pp. 1811-1819, 2005.

[8] S. Moaveni, Finite Element Analysis Theory and Application with ANSYS, Pearson, London, UK, 2011.

[9] E. Madenci and I. Guven, The Finite Element Method and Applications in Engineering Using ANSYS, Springer, Berlin, Germany, 2015.
[10] D.-C. Feng and J.-Y. Wu, "Improved displacement-based Timoshenko beam element with enhanced strains," Journal of Structural Engineering, vol. 146, no. 3, Article ID 04019221, 2020.

[11] S. J. Pantazopoulou, "Low-order interpolation functions for curved beams," Journal of Engineering Mechanics, vol. 118, no. 2, pp. 329-350, 1992.

[12] A. H. Sheikh, "New concept to include shear deformation in a curved beam element," Journal of Structural Engineering, vol. 128, no. 3, pp. 406-410, 2002.

[13] P. Raveendranath, G. Singh, and G. Venkateswara Rao, “A three-noded shear-flexible curved beam element based on coupled displacement field interpolations," International Journal for Numerical Methods in Engineering, vol. 51, no. 1, pp. 85-101, 2001.

[14] A. Benlemlih and M. El Alami El Ferricha, "A mixed finite element method for the arch problem," Applied Mathematical Modelling, vol. 26, no. 1, pp. 17-36, 2002.

[15] R. K. Kapania and J. Li, "A formulation and implementation of geometrically exact curved beam elements incorporating finite strains and finite rotations," Computational Mechanics, vol. 30, no. 5-6, pp. 444-459, 2003.

[16] J.-S. Wu and L.-K. Chiang, "Free vibration analysis of arches using curved beam elements," International Journal for $\mathrm{Nu}$ merical Methods in Engineering, vol. 58, no. 13, pp. 1907-1936, 2003.

[17] Z. Q. Wan, S. R. Li, and H. W. Ma, "Geometrically nonlinear analysis of functionally graded Timoshenko curved beams with variable curvatures," Advances in Materials Science and Engineering, vol. 2019, Article ID 6204145, 10 pages, 2019.

[18] J. C. Simo and L. Vu-Quoc, "A three-dimensional finite-strain rod model. Part II: computational aspects," Computer Methods in Applied Mechanics and Engineering, vol. 58, no. 1, pp. 79-116, 1986.

[19] A. Ibrahimbegović, "On finite element implementation of geometrically nonlinear reissner's beam theory: three-dimensional curved beam elements," Computer Methods in Applied Mechanics and Engineering, vol. 122, no. 1-2, pp. 11-26, 1995.

[20] A. F. Saleeb and T. Y. Chang, "On the hybrid-mixed formulation of C0 curved beam elements," Computer Methods in Applied Mechanics and Engineering, vol. 60, no. 1, pp. 95-121, 1987.

[21] H.-S. Ryu and H.-C. Sin, "Curved beam elements based on strain fields," Communications in Numerical Methods in Engineering, vol. 12, no. 11, pp. 767-773, 1996.

[22] C.-N. Chen, "DQEM analysis of in-plane vibration of curved beam structures," Advances in Engineering Software, vol. 36, no. 6, pp. 412-424, 2005.

[23] P. Litewka and J. Rakowski, "An efficient curved beam finite element," International Journal for Numerical Methods in Engineering, vol. 40, no. 14, pp. 2629-2652, 1997.

[24] P. Raveendranath, G. Singh, and B. Pradhan, "A two-noded locking-free shear flexible curved beam element," International Journal for Numerical Methods in Engineering, vol. 44, no. 2, pp. 265-280, 1999.

[25] G. Chen, F. Ma, G. Hao, and W. Zhu, "Modeling large deflections of initially curved beams in compliant mechanisms using chained beam constraint model," Journal of Mechanisms and Robotics, vol. 11, no. 1, Article ID 011002, 2019.

[26] K. Wu, G. Zheng, and G. Hao, "Efficient spatial compliance analysis of general initially curved beams for mechanism synthesis and optimization," Mechanism and Machine Theory, vol. 162, Article ID 104343, 2021. 
[27] E. Reissner, "On finite deformations of space-curved beams," ZAMP Zeitschrift for angewandte Mathematik und Physik, vol. 32, no. 6, pp. 734-744, 1981.

[28] J. H. Argyris, H. Balmer, J. S. Doltsinis et al., "Finite element method-the natural approach," Computer Methods in Applied Mechanics and Engineering, vol. 17-18, pp. 1-106, 1979.

[29] H. Stolarskl, T. Belytschko, and N. Carpenter, "Bending and shear mode decomposition in $\mathrm{C}^{\circ}$ structural elements," Journal of Structural Mechanics, vol. 11, no. 2, pp. 153-176, 1983.

[30] K.-J. Bathe, Finite Element Procedures, Prentice-Hall, Hoboken, NJ, USA, 2006. 